\title{
Quinine Dimers Are Potent Inhibitors of the Plasmodium falciparum Chloroquine Resistance Transporter and Are Active against Quinoline-Resistant $P$. falciparum
}

\author{
Christine A. Hrycyna, ${ }^{* \dagger}{ }^{\dagger}$ Robert L. Summers, ${ }^{\ddagger}$ Adele M. Lehane, ${ }^{\ddagger}$ Marcos M. Pires, ${ }^{\dagger}$ Hilda Namanja, ${ }^{\dagger}$
} Kelsey Bohn, ${ }^{\dagger}$ Jerrin Kuriakose, ${ }^{\dagger}$ Michael Ferdig, ${ }^{\S}$ Philipp P. Henrich, ${ }^{\|}$David A. Fidock, ${ }^{\|, \perp}$ Kiaran Kirk, ${ }^{\ddagger}$ Jean Chmielewski, ${ }^{* \dagger}$ and Rowena E. Martin**,

${ }^{\dagger}$ Department of Chemistry, Purdue University, West Lafayette, Indiana 47907, United States

${ }^{\ddagger}$ Research School of Biology, The Australian National University, Canberra, Australian Capital Territory 0200, Australia

${ }^{\S}$ Department of Biological Sciences, University of Notre Dame, Notre Dame, Indiana 46556, United States

"Department of Microbiology and Immunology, Columbia University, New York, New York 10027, United States

${ }^{\perp}$ Division of Infectious Diseases, Department of Medicine, Columbia University, New York, New York 10027, United States

Supporting Information

ABSTRACT: Chloroquine (CQ) resistance in the human malaria parasite Plasmodium falciparum is primarily conferred by mutations in the "chloroquine resistance transporter" (PfCRT). The resistance-conferring form of PfCRT (PfCRT ${ }^{\mathrm{CQR}}$ ) mediates $\mathrm{CQ}$ resistance by effluxing the drug from the parasite's digestive vacuole, the acidic compartment in which CQ exerts its antiplasmodial effect. PfCRT ${ }^{\mathrm{CQR}}$ can also decrease the parasite's susceptibility to other quinoline drugs, including the current antimalarials quinine and amodiaquine. Here we describe interactions between $\mathrm{PfCRT}^{\mathrm{CQR}}$ and a series of dimeric quinine molecules using a Xenopus laevis oocyte system for the heterologous expression of PfCRT and using an assay that detects the drug-associated

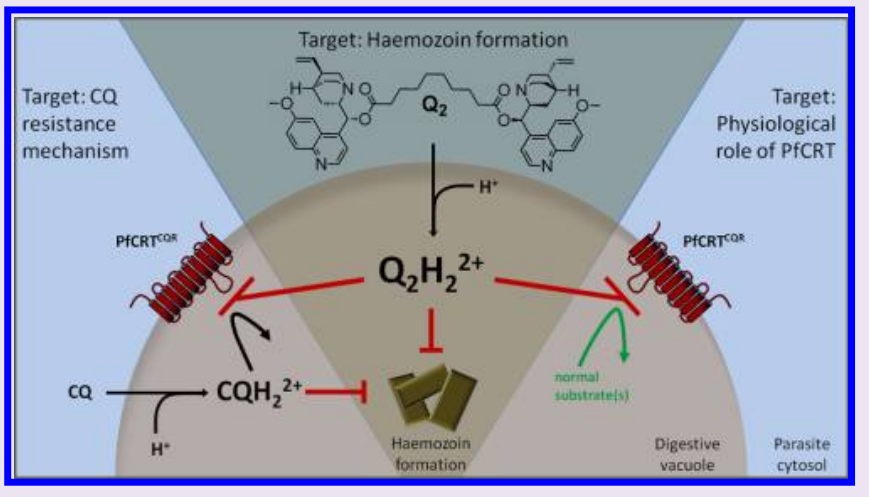
efflux of $\mathrm{H}^{+}$ions from the digestive vacuole in parasites that harbor different forms of PfCRT. The antiplasmodial activities of dimers 1 and $\mathbf{6}$ were also examined in vitro (against drug-sensitive and drug-resistant strains of $P$. falciparum) and in vivo (against drug-sensitive $P$. berghei). Our data reveal that the quinine dimers are the most potent inhibitors of PfCRT ${ }^{\mathrm{CQR}}$ reported to date. Furthermore, the lead compounds (1 and 6) were not effluxed by PfCRT ${ }^{\mathrm{CQR}}$ from the digestive vacuole but instead accumulated to very high levels within this organelle. Both 1 and $\mathbf{6}$ exhibited in vitro antiplasmodial activities that were inversely correlated with CQ. Moreover, the additional parasiticidal effect exerted by $\mathbf{1}$ and $\mathbf{6}$ in the drug-resistant parasites was attributable, at least in part, to their ability to inhibit PfCRT ${ }^{\mathrm{CQR}}$. This highlights the potential for devising new antimalarial therapies that exploit inherent weaknesses in a key resistance mechanism of $P$. falciparum.

$\mathrm{T}$ he human parasite Plasmodium falciparum causes the most severe form of malaria and is prevalent in nearly 100 countries, placing almost half the world's population at risk of acquiring the disease. ${ }^{1}$ The emergence of drug-resistant strains of $P$. falciparum has severely limited our ability to treat malaria. ${ }^{2}$ Strains resistant to the quinoline drugs chloroquine (CQ) and amodiaquine are widespread, ${ }^{3}$ and resistance to the current mainstays of malaria treatment (the artemisinin-based therapies) has recently been identified along the western Cambodia-Thailand border. ${ }^{4,5}$ The prevalence of multiple types of drug-resistant $P$. falciparum strains has created a tremendous and pressing need for new antimalarial drugs. Ideally, new drugs would not only act as potent antimalarial agents but would also be refractory to the known mechanisms of drug resistance.
The quinoline drugs $\mathrm{CQ}$ amodiaquine, and quinine are weak bases that exert their antimalarial effect, at least in part, by accumulating via "weak-base trapping" within the acidic environment of the parasite's digestive vacuole (DV). ${ }^{6}$ Here they are thought to prevent the conversion of toxic heme monomers (released from the parasite's digestion of host hemoglobin) into the inert crystal hemozoin. ${ }^{7,8}$ Resistance to $\mathrm{CQ}$ amodiaquine, and quinine has been correlated with a reduction in the accumulation of these drugs in the DV. ${ }^{9,10}$ This phenomenon is thought to be due to an increase in the efflux of the drug from the DV, a decrease in its uptake into the

Received: December 5, 2013

Accepted: December 26, 2013

Published: December 26, 2013 


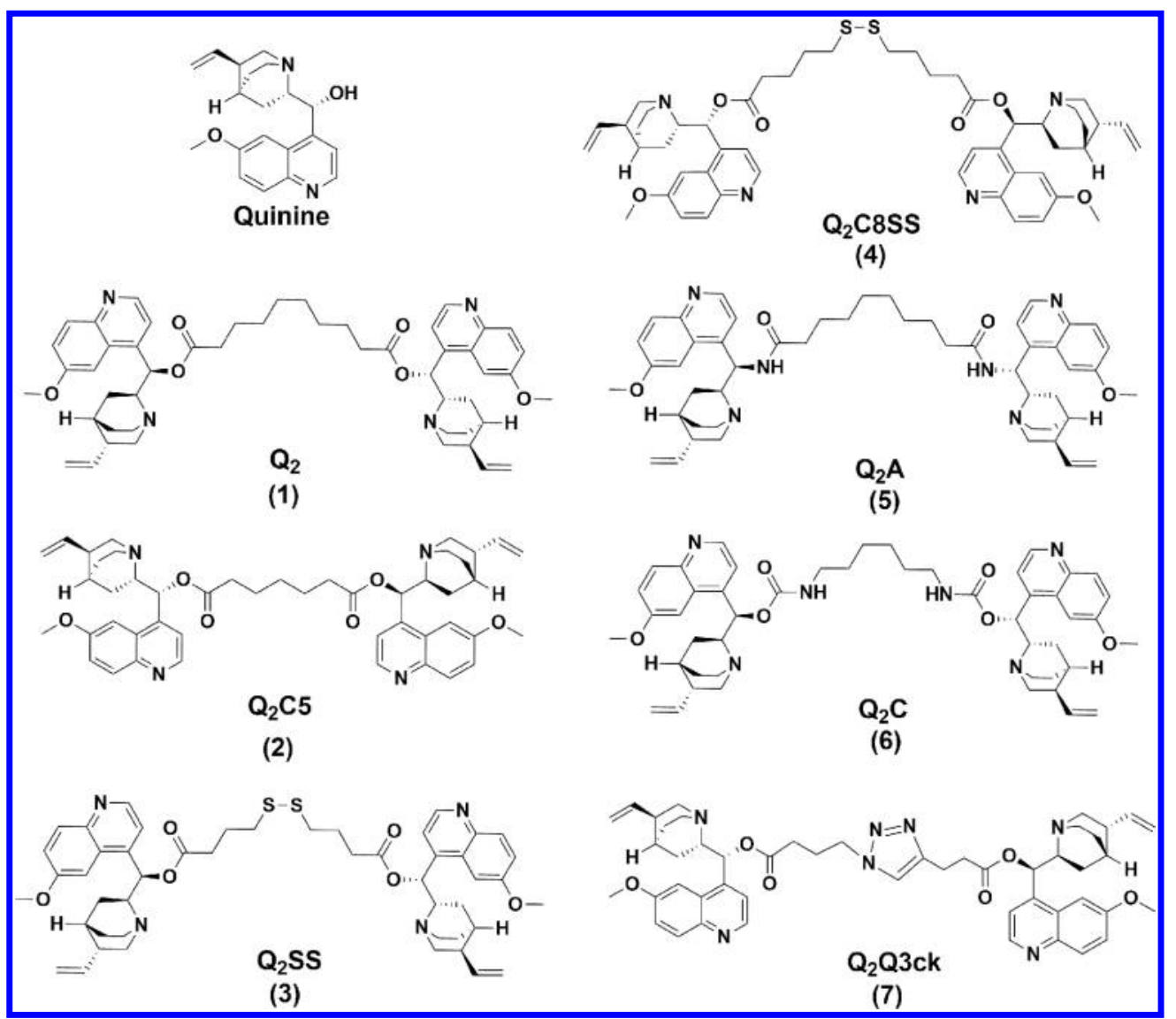

Figure 1. Structures of quinine and quinine homodimers.

DV, or a combination of both. ${ }^{11}$ The genetics of quinoline resistance in P. falciparum is complex and involves several genes encoding membrane transport proteins. These transporters include the chloroquine resistance transporter (PfCRT), the multidrug resistance transporter 1 (PfMDR1), and the multidrug resistance-associated protein 1 (PfMRP1). ${ }^{10,12-15}$

PfCRT is the best studied of these proteins and is located in the membrane of the DV. ${ }^{10,16,17}$ It is now widely accepted that mutations in PfCRT are the primary determinant of $\mathrm{CQ}$ resistance in $P$. falciparum and that they can also modulate the parasite's sensitivity to other quinolines. ${ }^{11,13,18}$ The key mutation associated with CQ resistance is the replacement of the lysine $(\mathrm{K})$ at position 76 with threonine $(\mathrm{T})$, resulting in the loss of a positive charge from the putative substrate-binding site of the transporter. ${ }^{19,20}$ The variant of PfCRT habored by the CQ-resistant (CQR) strain Dd2 (PfCRT ${ }^{\mathrm{CQR}}$ ) contains the crucial K76T mutation as well as seven other mutations. When expressed in the plasma membrane of Xenopus laevis oocytes, PfCRT $^{\mathrm{CQR}}$ mediates the transport of $C Q$, whereas the $\mathrm{CQ}$ sensitive (CQS) form of the protein (PfCRT ${ }^{\mathrm{CQS}}$ ) does not. ${ }^{12}$ These data are consistent with the hypothesis that PfCRT ${ }^{\mathrm{CQR}}$ confers CQ resistance by exporting the drug out of the DV, away from its primary site of action. It is important to note that attempts to generate transfectant parasite lines in which pfcrt is knocked out have been unsuccessful, and efforts to silence the expression of its ortholog in $P$. berghei have also failed. ${ }^{17,21}$ Hence, quite apart from its role in mediating CQ resistance, PfCRT fulfills an essential physiological function in the parasite. What this role might be remains unknown.

The oocyte system allows interactions between PfCRT ${ }^{\mathrm{CQR}}$ and candidate antiplasmodial compounds to be studied directly and in isolation, without confounding effects such as the binding of drug to heme or to other targets within the parasite. For example, a number of compounds, including quinine and the CQ "resistance reverser" verapamil, have been shown to inhibit the PfCRT ${ }^{\mathrm{CQR}}$-mediated uptake of $\left[{ }^{3} \mathrm{H}\right] \mathrm{CQ}$ into oocytes in a concentration-dependent manner. ${ }^{12,22}$ Further evidence of the ability of PfCRT ${ }^{\mathrm{CQR}}$ to interact with drugs has been obtained using a fluorescence-based assay that detects the drugassociated efflux of $\mathrm{H}^{+}$ions from the DV of parasites. Application of this method to parasite lines that were isogenic except for their $p f c r t$ allele (which encoded a CQS or CQR form of the protein) revealed that $\mathrm{PfCRT}^{\mathrm{CQR}}$ mediates the transport of $\mathrm{CQ}$ quinine, and several other antimalarial agents in situ. ${ }^{23-25}$ Moreover, measurements of $\left[{ }^{3} \mathrm{H}\right]$ quinine efflux from the same parasite lines revealed a verapamil-sensitive component attributable to PfCRT ${ }^{\mathrm{CQR}}{ }^{26}$ Taken together, these data suggest that quinine is also a substrate of PfCRT ${ }^{\mathrm{CQR}}$.

A number of CQ-related compounds are equally active against CQS and CQR strains. ${ }^{11,27,28}$ One explanation for this phenomenon is that these compounds are not recognized or translocated by PfCRT ${ }^{\mathrm{CQR}}$ and thereby evade the resistance mechanism imparted by the mutant protein. ${ }^{11}$ Examples include analogues of CQ in which the side chain has been modified as well as the antimalarial drug piperaquine (currently used in combination with dihydroartemisinin). ${ }^{29}$ Piperaquine consists of two 7-chloroquinoline nuclei coupled via an aminecontaining side chain and can therefore be considered a dimer of CQ. Changes to PfCRT or PfMDR1 that confer reduced susceptibilities to other quinolines have little or no effect on the parasite's sensitivity to piperaquine, ${ }^{30}$ and piperaquine does not display cross-resistance with $\mathrm{CQ}$ in field isolates. ${ }^{31}$ Moreover, 
when tested in the oocyte system, piperaquine lacked the ability to inhibit the transport of $\left[{ }^{3} \mathrm{H}\right] \mathrm{CQ}$ via PfCRT ${ }^{\mathrm{CQR}}{ }^{12}$ Hence, several lines of evidence indicate that piperaquine bypasses the CQ resistance mechanism because it does not interact with CQR forms of PfCRT. On the other hand, in the case of the human homologues of PfMDR1 and PfMRP1, dimerization of substrate molecules produced inhibitors that bound more effectively to the transporter's substrate binding sites than the monomer. ${ }^{32-34}$ Given these findings, we hypothesized that dimers of other quinoline drugs may likewise either (1) escape the CQ resistance mechanism altogether, enabling the drug to accumulate within the DV of both CQS and CQR parasites (where it can exert its antihemozoin activity) or (2) block transport via PfCRT ${ }^{\mathrm{CQR}}$, which could also enable toxic levels of the drug to accumulate within the DV of both CQS and CQR parasites. In the latter scenario, the dimerized drug may exert a second antiplasmodial effect in CQR parasites as a consequence of inhibiting the normal function the transporter. Here we report the activities of dimeric quinine agents against CQS and CQR strains of $P$. falciparum as well as a detailed investigation of their interactions with PfCRT ${ }^{\mathrm{CQR}}$.

\section{RESULTS AND DISCUSSION}

Dimeric Quinine Molecules Inhibited CQ Transport via PfCRT ${ }^{\mathrm{CQR}}$ in X. laevis Oocytes. A series of quinine dimers containing several different tethers linked via ester, carbamate, or amide bonds were designed and synthesized (Figure 1). The compounds included the ester-linked molecules 1-4, the amide-containing 5 , the carbamate-linked dimer $\mathbf{6}$, and the triazole-containing "click" dimer 7. The esters (1-4) were synthesized from quinine and the corresponding diacid. The amide 5 was prepared from aminoquinine ${ }^{35}$ and sebacic acid. The carbamate 6 was prepared from quinine, $p$-nitrophenylchloroformate, and 1,6-hexadiamine. Compound 7 was prepared from alkyne- and azide-modified quinine derivatives as previously described. ${ }^{36}$ The compounds were purified by either flash silica chromatography or reverse phase HPLC and were analyzed by ${ }^{1} \mathrm{H}$ NMR, analytical HPLC, and mass spectrometry.

The ability of the dimeric quinine molecules to inhibit the transport of $\left[{ }^{3} \mathrm{H}\right] \mathrm{CQ}$ via the $\mathrm{Dd} 2$ version of PfCRT ${ }^{\mathrm{CQR}}$ was evaluated using the Xenopus oocyte system. ${ }^{12}$ In this assay, PfCRT is expressed in the oocyte plasma membrane, and the direction of $\left[{ }^{3} \mathrm{H}\right] \mathrm{CQ}$ transport is from the extracellular medium ( $\mathrm{pH}$ 6.0) into the oocyte cytosol ( $\mathrm{pH}$ 7.2), which corresponds to the efflux of CQ from the acidic DV ( $\mathrm{pH} 5$ to 5.5) into the parasite cytosol ( $\mathrm{pH} 7.3$ ). In an initial experiment in which the quinine dimers were assessed at an extracellular concentration of $100 \mu \mathrm{M}$, the compounds either abolished CQ transport via PfCRT $^{C Q R}$ (e.g., 5 and 6) or caused significant reductions in the PfCRT ${ }^{\mathrm{CQR}}$-mediated uptake of CQ (e.g., 1 and 3; Figure 2; $P<0.001$, ANOVA). None of the compounds affected the diffusion of CQ into oocytes expressing PfCRT ${ }^{\mathrm{CQS}}$ (Figure 2; $P$ $>0.05$, ANOVA). Furthermore, all of the compounds reduced the accumulation of $\left[{ }^{3} \mathrm{H}\right] \mathrm{CQ}$ in oocytes expressing PfCRT ${ }^{\mathrm{CQR}}$ to levels significantly below those measured in the presence of verapamil $(100 \mu \mathrm{M})$ or quinine $(100 \mu \mathrm{M}$ or $200 \mu \mathrm{M})$ (Figure 2; $P<0.001$, ANOVA). The latter result indicates that the potency of the quinine dimers against $P f C R T^{C Q R}$ is not simply due to a doubling in the amount of quinine in the reaction buffer but is a consequence, at least in part, of an enhanced ability to inhibit PfCRT ${ }^{\mathrm{CQR}}$.

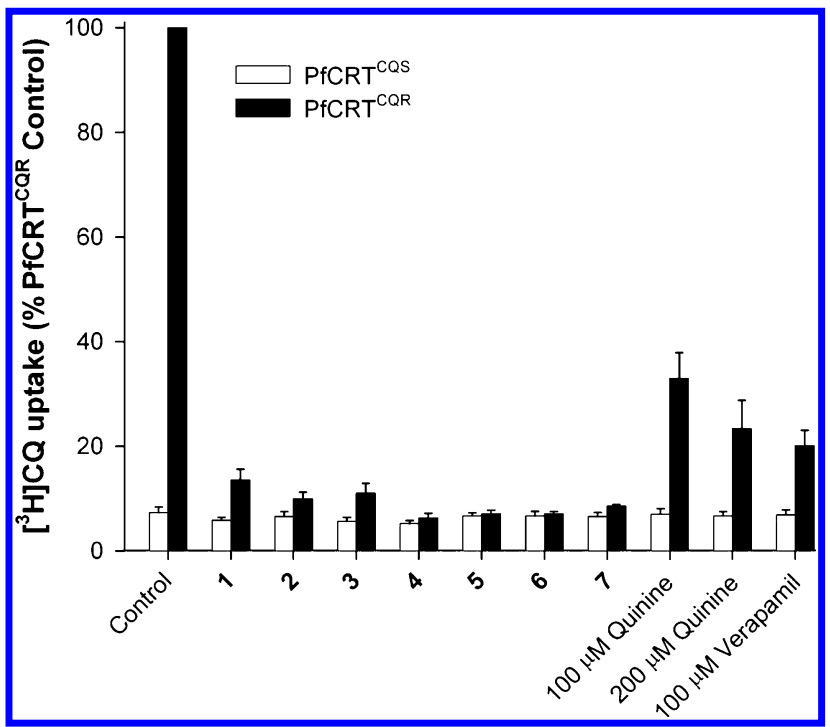

Figure 2. The effect of dimeric quinine compounds on PfCRT ${ }^{\mathrm{CQR}}$. mediated transport of $\mathrm{CQ}$ in $X$. laevis oocytes. $\left[{ }^{3} \mathrm{H}\right] \mathrm{CQ}$ uptake into oocytes expressing Dd2 PfCRT ${ }^{\mathrm{CQR}}$ (black bars) or D10 PfCRT ${ }^{\mathrm{CQS}}$ (white bars) in the presence of unlabeled dimeric compounds (100 $\mu \mathrm{M})$, quinine $(100$ or $200 \mu \mathrm{M})$, or verapamil $(100 \mu \mathrm{M})$. Uptake is shown as the mean + SEM from $n=4$ separate experiments, within which measurements were made from 10 oocytes per treatment.

An analysis of the concentration-dependence of the inhibition of PfCRT ${ }^{\mathrm{CQR}}$ by compounds $1,3,5$, and 6 yielded half-maximal inhibitory concentrations ( $\mathrm{IC}_{50}$ values) of 5.3, 5.8, 1.0, and $1.4 \mu \mathrm{M}$, respectively (Figure 3 and Table 1). These values are significantly lower than those measured previously for quinine $(48 \pm 6 \mu \mathrm{M})$, verapamil $(30 \pm 1 \mu \mathrm{M})$, and the antiretroviral drug saquinavir $(13 \pm 1 \mu \mathrm{M}){ }^{12,37}$ The $\mathrm{IC}_{50}$ values obtained for $\mathbf{5}$ and $\mathbf{6}$ were not significantly different from one another $(P>0.05)$ but were significantly lower than those measured for 1 and $3(P<0.001)$. Compounds 5 and 6 are therefore the most potent PfCRT ${ }^{\mathrm{CQR}}$ inhibitors described to date, with 9.3- and 13.0-fold increases in potency relative to saquinavir, respectively. It should be noted that the low micromolar concentrations of the compounds used here to inhibit PfCRT ${ }^{\mathrm{CQR}}$ are physiologically relevant, given that when present in the extracellular solution at submicromolar concentrations, these tetrabasic compounds are expected to accumulate to high micromolar and even millimolar concentrations within the DV via weak-base trapping.

An assessment of cellular toxicity (using $24 \mathrm{~h}$ MTT assays performed on the human cell lines MCF-7 and MCF-7/DX-1; data not shown) revealed that compound 5 possesses significant cytotoxic potential, and previous work has indicated that compound 3 is likely to undergo reduction in cytosolic environments. $^{38}$ All subsequent analyses were therefore performed with compounds 1 and 6 .

Compounds 1 and 6 Were Inhibitors but Not Substrates of PfCRT ${ }^{\mathrm{CQR}}$ in Situ. Compounds that inhibit $\mathrm{PfCRT}^{\mathrm{CQR}}$ may themselves be substrates of the transporter, which could be problematic if this leads to cross-resistance with CQ. We therefore employed a fluorescence-based assay to resolve the nature of the interactions between PfCRT ${ }^{\mathrm{CQR}}$ and compounds 1 and $\mathbf{6}$ in parasitized red blood cells. This method entails monitoring the drug-associated efflux of $\mathrm{H}^{+}$ions from the $\mathrm{DV}$ using a fluorescent $\mathrm{pH}$-sensitive probe targeted specifically to this compartment. ${ }^{23,24}$ A drug-induced $\mathrm{H}^{+}$leak 


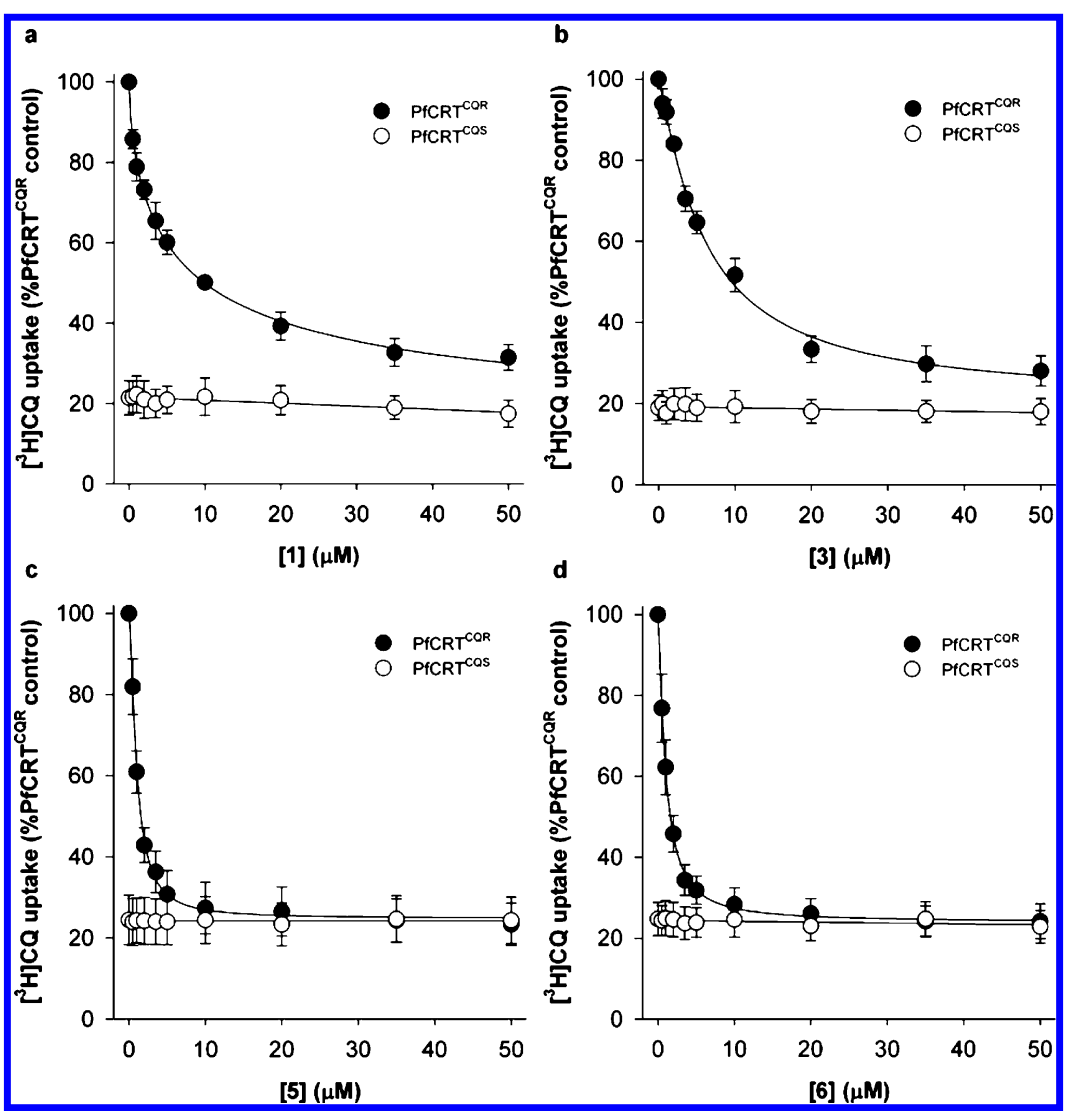

Figure 3. Concentration-dependent inhibition of CQ transport via PfCRT ${ }^{\mathrm{CQR}}$. Concentration-dependent effects of (a) 1, (b) 3, (c) 5, and (d) 6 on the uptake of $\left[{ }^{3} \mathrm{H}\right] \mathrm{CQ}$ into oocytes expressing Dd2 PfCRT ${ }^{\mathrm{CQR}}$ (solid circles) or D10 PfCRT ${ }^{\mathrm{CQS}}$ (open circles). Uptake is shown as the mean \pm SEM from $n=4$ or 5 separate experiments, within which measurements were made from 10 oocytes per treatment.

Table 1. IC $_{50}$ Values for the Inhibition of PfCRT ${ }^{\mathrm{CQR}}$ Mediated CQ Transport by Quinine Dimers

$\begin{array}{lc}\text { compound } & \mathrm{IC}_{50}(\mu \mathrm{M})^{a} \\ \text { verapamil } & 30 \pm 3^{12} \\ \text { quinine } & 48 \pm 6^{12} \\ \mathbf{1} & 5.3 \pm 0.3 \\ \mathbf{3} & 5.8 \pm 0.5 \\ \mathbf{5} & 1.0 \pm 0.1 \\ \mathbf{6} & 1.4 \pm 0.2\end{array}$

${ }^{a}$ PfCRT ${ }^{\mathrm{CQR}}$-mediated CQ transport was calculated by subtracting the uptake measured in oocytes expressing D10 PfCRT ${ }^{\mathrm{CQS}}$ from that in oocytes expressing Dd2 PfCRT ${ }^{\mathrm{CQR}}$. The half-maximal inhibitory concentrations ( $\mathrm{IC}_{50}$ values) were derived by least-squares fit of the equation $Y=Y_{\min }+\left[\left(Y_{\max }-Y_{\min }\right) /\left(1+\left([\right.\right.\right.$ inhibitor $\left.\left.\left.] / \mathrm{IC}_{50}\right) C\right)\right]$ where $Y$ is $\mathrm{PfCRT}^{\mathrm{CQR}}$-mediated $\mathrm{CQ}$ transport, $Y_{\min }$ and $Y_{\max }$ are the minimum and maximum values of $Y$, and $C$ is a constant. All values are mean \pm SEM from $n=4$ or 5 separate experiments, within which measurements were made from 10 oocytes per treatment. The data from which these values were obtained are shown in Figure 3.

arises when a weak-base drug enters the acidic DV in its unprotonated form and is effluxed in its protonated form (or in symport with $\mathrm{H}^{+}$). The outward leak of $\mathrm{H}^{+}$is detected as an increase in the rate of alkalinization of the DV upon inhibition of the V-type $\mathrm{H}^{+}$-ATPase with concanamycin A. ${ }^{23,24}$ The experiments described here were performed with the isogenic CQS and CQR pfcrt transfectant lines generated by Sidhu et al. $^{18}$ These parasite lines express either the wild-type $p f c r t$ allele $\left(\mathrm{C} 2^{\mathrm{GC} 03}\right)$ or the $p f c r t$ allele from the CQR strain $\mathrm{Dd} 2\left(\mathrm{C} 4^{\mathrm{Dd} 2}\right)$ or $7 \mathrm{G} 8\left(\mathrm{C6}^{7 \mathrm{G} 8}\right)$.
As shown in Figure 4, both quinine and CQ increased the rate of DV alkalinization in the CQR lines $(P<0.05$; paired $t$ test), and quinine was without effect in the CQS $\mathrm{C} 2^{\mathrm{GCO} 3}$ parasites $(P>0.05)$. These data replicate previous findings ${ }^{23-25}$ and are consistent with quinine and CQ being substrates of the $\mathrm{Dd} 2$ and $7 \mathrm{G} 8$ forms of PfCRT ${ }^{\mathrm{CQR}}$. By contrast, compounds 1 and 6 did not induce a $\mathrm{H}^{+}$leak from the DV in any of the three parasite lines, which indicates that these compounds are not substrates of PfCRT ${ }^{\mathrm{CQS}}$ or the $\mathrm{Dd} 2$ or $7 \mathrm{G} 8$ forms of PfCRT ${ }^{\mathrm{CQR}}$ (Figure 4a). Instead, compound 6 significantly decreased the rate of DV alkalinization in all three lines $(P<0.05)$, consistent with it accumulating to high concentrations in this organelle and exerting a buffering effect therein. ${ }^{23}$ Compound 1 caused a smaller decrease in the rate of DV alkalinization, which was significant in the $\mathrm{CQR}$ lines $(P<0.02)$ but not in $\mathrm{C}^{\mathrm{GC} 03}$ parasites $(P>0.05)$. Moreover, the efflux of $\mathrm{H}^{+}$induced by $\mathrm{CQ}$ $(2.5 \mu \mathrm{M})$ was abolished by the addition of 1 or $6(1 \mu \mathrm{M})$, consistent with these compounds inhibiting the $\mathrm{Dd} 2$ and 7G8 forms of PfCRT ${ }^{\mathrm{CQR}}$ in situ (Figure $4 \mathrm{~b}$ ).

Compounds 1 and 6 Were Stable in Vitro. The findings that (1) several different quinine dimers are more effective inhibitors of PfCRT than quinine and (2) quinine is transported by PfCRT ${ }^{\mathrm{CQR}}$ whereas compounds $\mathbf{1}$ and $\mathbf{6}$ are not suggest that the dimeric structure is responsible, at least in part, for the anti-PfCRT activities exhibited by the quinine dimers. It was therefore important to determine whether these compounds are likely to remain as dimers under the conditions of in vitro and in vivo assessments of antimalarial activity. The resistance of compounds 1 (ester-linked) and 6 (carbamatelinked) to plasma and cellular esterases was investigated by 


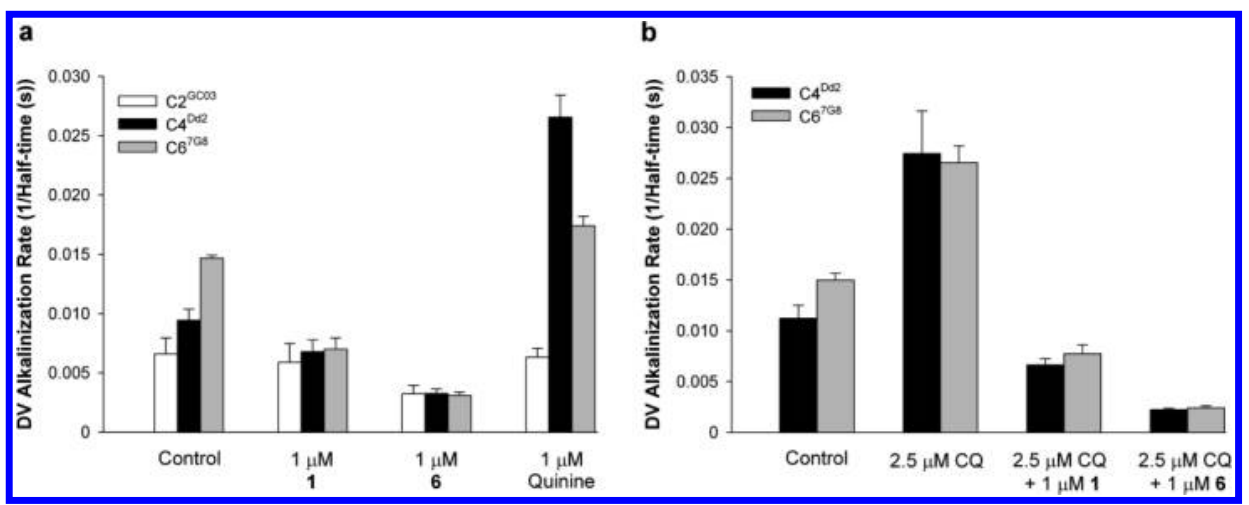

Figure 4. Concanamycin A-induced digestive vacuole (DV) alkalinization in pfcrt transfectant lines. The rate of concanamycin A-induced DV alkalinization in the $p f c r t$ transfectant lines $\mathrm{C}^{\mathrm{GC} 03}$ (CQS; white bars), $\mathrm{C} 4^{\mathrm{Dd} 2}$ (CQR; black bars), and $\mathrm{C}^{7 \mathrm{G} 8}$ (CQR; gray bars) was measured in the absence (solvent control) and presence of the compounds indicated. Compounds were added to the parasite suspensions 4 min before the addition of concanamycin A $(100 \mathrm{nM})$. The data are averaged from three independent experiments and shown + SEM: (a) 1, 6, and quinine were all added at a final concentration of $1 \mu \mathrm{M}$; (b) CQ was added at a concentration of $2.5 \mu \mathrm{M}$ in the presence or absence of $1(1 \mu \mathrm{M})$ and $6(1 \mu \mathrm{M})$.

testing their stability in the presence of human plasma or purified pig liver esterase (PLE), respectively (Table 2).

Table 2. Quinine Dimer Stability and Inhibition of $\boldsymbol{\beta}$ Hematin in Vitro

$\begin{array}{lccc}\text { compound } & t_{1 / 2}(\text { plasma })^{a}(\mathrm{~h}) & t_{1 / 2}(\text { esterase })^{a}(\mathrm{~h}) & \mathrm{IC}_{50}(\text { heme })^{b}(\mu \mathrm{M}) \\ \text { quinine } & & & 3.9 \pm 0.1 \\ 1 & >90 & 25 \pm 4 & 3.4 \pm 0.6 \\ 6 & >90 & >90 & 2.5 \pm 0.7\end{array}$

${ }^{a_{\text {The }}}$ The compounds $(40 \mu \mathrm{M})$ were incubated at $37{ }^{\circ} \mathrm{C}$ in a solution containing $55 \% \mathrm{vol} / \mathrm{vol}$ human plasma, $45 \% \mathrm{vol} / \mathrm{vol} \mathrm{PBS}$ ( $\mathrm{pH} 7.4$ ), and $0.3 \% \mathrm{vol} / \mathrm{vol} \mathrm{DMSO}$ or in 10 units of purified pig liver esterase in PBS. Monomeric quinine was detected by HPLC, and the half-lives were derived from fitted curves (Graph Pad Prism 4). ${ }^{b}$ Concentration of the compound that inhibited $50 \%$ of $\beta$-hematin production in vitro.

Compounds 1 and 6 were incubated in 55\% human plasma or with PLE (10 units) at $37{ }^{\circ} \mathrm{C}$, and the products were analyzed by HPLC to monitor the presence of the dimer and monomer. The half-life $\left(t_{1 / 2}\right)$ of each dimer was estimated by fitting curves to the resulting data. In human plasma, both 1 and 6 had $t_{1 / 2}$ values greater than $90 \mathrm{~h}$ (Table 2 ); after $90 \mathrm{~h}$, only $12 \%$ of 1 had reverted to monomer and compound 6 remained intact. Compounds 1 and 6 were also resistant to cleavage by PLE, with $t_{1 / 2}$ values of 25 and $>90 \mathrm{~h}$, respectively. The stabilities of $\mathbf{1}$ and $\mathbf{6}$ were also tested in an acidic buffer ( $\mathrm{pH} \mathrm{4.5,} \mathrm{to} \mathrm{mimic} \mathrm{the} \mathrm{acidic} \mathrm{environment} \mathrm{of} \mathrm{the} \mathrm{parasite} \mathrm{DV)}$ and in the medium used in the in vitro parasite proliferation assays. In both cases, neither compound reverted to the monomer over a $24 \mathrm{~h}$ period (data not shown). These data confirm that compounds $\mathbf{1}$ and $\mathbf{6}$ are stable under conditions that mimic key in vitro and in vivo assays.

Compounds 1 and 6 Inhibited $\beta$-Hematin Formation in Vitro. One mode by which quinine is thought to kill the parasite is the inhibition of heme detoxification in the DV. ${ }^{7,8}$ To determine whether the quinine dimers also possess antihemozoin activity, an in vitro colorimetric assay was performed to quantify the ability of $\mathbf{1}$ and $\mathbf{6}$ to inhibit the formation of synthetic hemozoin ( $\beta$-hematin). ${ }^{39}$ Both compounds were found to inhibit $\beta$-hematin formation in a dose-dependent manner, and the resulting $\mathrm{IC}_{50}$ values were comparable to that measured for quinine in the same experiment (Table 2).

Compounds 1 and 6 Were Active against DrugResistant $P$. falciparum Strains in Vitro. The antiplasmodial activities of compounds $\mathbf{1}$ and $\mathbf{6}$ were evaluated in vitro against red blood cells infected with field-derived $P$. falciparum strains. These included the CQS strain HB3 (isolated in Honduras and carrying PfCRT ${ }^{\mathrm{CQS}}$ ) and the CQR strains Dd2 (isolated in Indochina and carrying the $\mathrm{Dd} 2$ version of PfCRT ${ }^{\mathrm{CQR}}$ ) and FCB and P31 (both isolated in South East Asia and carrying the $\mathrm{K} 1$ version of $\left.\mathrm{PfCRT}{ }^{\mathrm{CQR}}\right)$. Incorporation of $\left[{ }^{3} \mathrm{H}\right]$-hypoxanthine into parasite nucleic acids was used as a measure of parasite growth, and each compound (as well as quinine) was tested at a range of concentrations. ${ }^{40-43}$ The resulting $\mathrm{IC}_{50}$ values are presented in Table 3. Quinine was significantly less active against the CQR strains than against the CQS strain $(P<0.05)$, with the fold-differences in the quinine $\mathrm{IC}_{50}$ values between the HB3 clone and the CQR strains being 5 (P31), 3.3 (FCB), and 1.7 (Dd2). Both 1 and 6 inhibited the proliferation of $P$. falciparum with $\mathrm{IC}_{50}$ values in the nanomolar range, although neither compound was more effective than quinine against the

Table 3. In Vitro Antiplasmodial Activities of Quinine and Compounds 1 and 6 against Field-Derived Drug-Sensitive and DrugResistant Strains of $P$. falciparum

\begin{tabular}{cccc} 
& \multicolumn{2}{c}{ parasite strain, $\mathrm{IC}_{50}(\mathrm{nM})^{a}$} \\
\cline { 2 - 4 } compound & $\mathrm{Dd} 2$ & $\mathrm{FCB}$ & $\mathrm{P} 31$ \\
quinine & $187.7 \pm 28.9$ & $354.7 \pm 17.8$ & $535.3 \pm 40.8$ \\
1 & $132.6 \pm 23.8$ & $91.1 \pm 5.6$ & $186.6 \pm 12.4$ \\
6 & $49.2 \pm 6.2$ & $32.0 \pm 5.4$ & $232.1 \pm 31.1$
\end{tabular}

${ }^{a_{\text {The }}}$ half-maximal inhibitory concentrations ( $\mathrm{IC}_{50}$ values) were determined using a $\left[{ }^{3} \mathrm{H}\right]$-hypoxanthine incorporation assay as described in the Methods. The data is shown as mean \pm SEM from 4 separate experiments performed on different days. P. falciparum strains: drug-sensitive strain: HB3 (Honduras); drug-resistant strains: P31 (South East Asia), FCB (South East Asia), Dd2 (Indochina). 
Table 4. In Vitro Antiplasmodial Activities of Quinine, CQ and Compounds 1 and 6 against Isogenic Drug-Sensitive and DrugResistant Strains of P. falciparum

\begin{tabular}{|c|c|c|c|}
\hline \multirow[b]{2}{*}{ compound } & \multicolumn{3}{|c|}{ parasite strain, $\mathrm{IC}_{50}(\mathrm{nM})^{a}$} \\
\hline & $\mathrm{C} 2^{\mathrm{GC} 03}$ & $\mathrm{C} 4^{\mathrm{Dd} 2}$ & $\mathrm{C} 6^{7 \mathrm{G} 8}$ \\
\hline quinine & $56 \pm 3$ & $18.4 \pm 0.9(0.009)$ & $15.9 \pm 0.7(0.009)$ \\
\hline CQ & $12.4 \pm 1.7$ & $31.0 \pm 1.9(0.01)$ & $26.9 \pm 3.7(0.03)$ \\
\hline monodesethyl-CQ & $31.4 \pm 1.5$ & $>1500^{b}$ & $855 \pm 186(0.05)$ \\
\hline 1 & $487 \pm 23$ & $209 \pm 14(0.002)$ & $239 \pm 38(0.01)$ \\
\hline 6 & $286 \pm 14$ & $75 \pm 11(<0.001)$ & $82 \pm 12(<0.001)$ \\
\hline
\end{tabular}

${ }^{a}$ The half-maximal inhibitory concentrations ( $\mathrm{IC}_{50}$ values) for the compounds indicated in the pfcrt transfectant lines generated by Sidhu et al. ${ }^{18}$ The data is shown as mean \pm SEM from three separate experiments performed on different days. $P$ values from paired $t$-test comparisons with $\mathrm{C}_{2}{ }^{\mathrm{GC} 03}$ are shown in parentheses. ${ }^{b}$ Highest concentration tested.

CQS strain. However, in contrast with what was observed for quinine, the efficacy of compound 6 against the $\mathrm{Dd} 2$ and FCB parasites was considerably greater than that measured in the CQS strain $(P<0.05)$, and compound 1 was more potent against all three CQR strains than against the HB3 clone $(P<$ $0.05)$. Moreover, the $\mathrm{IC}_{50}$ values determined for $\mathbf{1}$ and $\mathbf{6}$ were lower than those obtained for quinine in all three CQR strains $(P<0.05)$. These in vitro data demonstrate that compounds 1 and 6 are potent antiplasmodial agents that have activities that are inversely correlated with those of quinine and CQ in fieldderived strains of $P$. falciparum.

These findings were investigated further by measuring the antiplasmodial activities of compounds $\mathbf{1}$ and $\mathbf{6}$ against the $\mathrm{C} 2^{\mathrm{GC} 03}, \mathrm{C} 4^{\mathrm{Dd} 2}$, and $\mathrm{C}^{7 \mathrm{G} 8}$ parasite lines. The efficacies of quinine, $\mathrm{CQ}$ and monodesethyl-CQ (the main metabolite of CQ) were also tested. Consistent with previous reports, the $\mathrm{IC}_{50}$ values determined for CQ and monodesethyl-CQ were higher in the CQR lines than in the CQS counterpart (Table 4; $P<0.05$, paired $t$-tests). ${ }^{18}$ The opposite pattern was observed for 1,6 , and quinine, with the $\mathrm{C} 4^{\mathrm{Dd} 2}$ and $\mathrm{C} 6^{7 \mathrm{G} 8}$ parasites being 2-4 times more susceptible than the $\mathrm{C}_{2}{ }^{\mathrm{GC} 03}$ line to these compounds (Table 4; $P<0.05$, paired $t$-tests). Given that the transfectant lines differ only in the version of the pfcrt gene expressed, these data indicate that the enhanced potency of 1 and 6 against the CQR parasites is, at least in part, due to an interaction with PfCRT ${ }^{\mathrm{CQR}}$. By binding to PfCRT ${ }^{\mathrm{CQR}}$ with high affinity, these drugs would block the normal physiological function of the transporter (which is known to be essential for the survival of the parasite $)^{17,21}$ and thereby exert an additional killing effect. Hence, the quinine dimers possess both antihemozoin and anti-PfCRT ${ }^{\mathrm{CQR}}$ activities. The higher $\mathrm{IC}_{50}$ values obtained for $\mathbf{1}$ and $\mathbf{6}$ relative to quinine in both the fieldderived and transgenic parasites may be due to the dimers being less effective inhibitors of hemozoin formation in vivo or other (as yet undefined) targets of quinine.

Compounds $\mathbf{1}$ and $\mathbf{6}$ were potent inhibitors of CQ transport via $\mathrm{PfCRT}^{\mathrm{CQR}}$ in the oocyte system as well as in the $\mathrm{H}^{+}$-leak assay. We therefore examined their ability to act as $\mathrm{CQ}$ resistance reversers in the $\mathrm{C} 2{ }^{\mathrm{GC} 03}, \mathrm{C}^{\mathrm{Dd} 2}$, and $\mathrm{C}^{7 \mathrm{G} 8}$ lines. $\mathrm{CQ}$ growth assays were undertaken in the absence or presence of a very low concentration $(62.5 \mathrm{nM})$ of $\mathbf{1 , 6}$, or verapamil, and the resulting $\mathrm{CQ} \mathrm{IC}_{50}$ values are presented in Figure 5. At this low concentration, neither verapamil nor compound 1 exerted CQ resistance-reversing activity, whereas compound 6 chemosensitized both of the CQR lines to CQ $(P \leq 0.05)$. This result is consistent with our findings that compound 6 is a much more potent inhibitor of $\mathrm{PfCRT}^{\mathrm{CQR}}$-mediated $\mathrm{CQ}$ transport $\left(\mathrm{IC}_{50}\right.$ of $\sim 1.4 \mu \mathrm{M}$ ) than either 1 ( $\mathrm{IC}_{50}$ of $\sim 5.3$ $\mu \mathrm{M}$ ) or verapamil $\left(\mathrm{IC}_{50}\right.$ of $\left.\sim 30 \mu \mathrm{M}\right)$, as well as with the finding

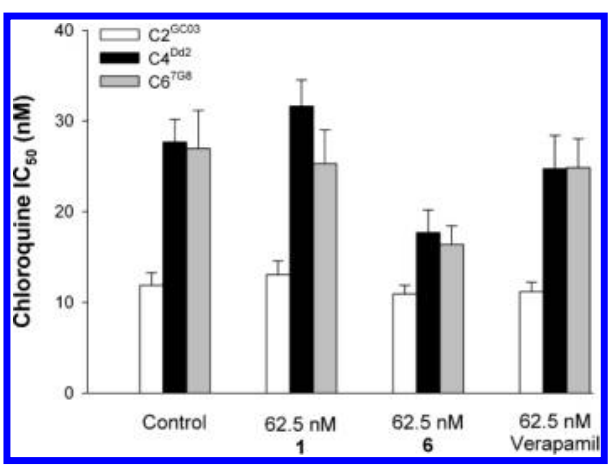

Figure 5. Growth inhibition of pfcrt transfectant lines by CQ. CQ $\mathrm{IC}_{50}$ values for the $p f c r t$ transfectant lines $\mathrm{C}^{\mathrm{GC} 03}$ (CQS; white bars), $\mathrm{C}^{\mathrm{Dd} 2}$ (CQR; black bars), and $\mathrm{C6}^{7 \mathrm{G} 8}$ ( $\mathrm{CQR}$; gray bars) in the presence and absence of 1,6 , or verapamil (all added at a final concentration of 62.5 $\mathrm{nM})$. The data are averaged from three independent experiments and shown + SEM

that in all strains compound 6 decreased the rate of (concanamycin A-induced) alkalinization of the DV to a greater extent than compound $\mathbf{1}$ (Figure 4a), an observation attributed to compound $\mathbf{6}$ accumulating to higher levels than compound $\mathbf{1}$ in the DV and hence exerting a stronger buffering effect.

Taken together, these data suggest that the CQ resistance mechanism could be overcome by administering $\mathbf{6}$ (or a similar compound) in combination with CQ (or other quinoline antiplasmodials that display cross-resistance with $\mathrm{CQ}$ for example, amodiaquine and pyronaridine). In such a therapy, both 6 and the partner quinoline would possess antihemozoin activity against the CQS strains. In CQR strains, 6 would exert an antihemozoin effect while, at the same time, blocking the efflux of the partner quinoline via PfCRT ${ }^{\mathrm{CQR}}$, thereby enhancing the antiplasmodial action of this compound. The finding that compound 6 blocks the flux of CQ via PfCRT ${ }^{\mathrm{CQR}}$ also raises the possibility that it may inhibit the normal transport function of PfCRT in CQR strains and that this too might contribute to its parasiticidal effect.

The finding that quinine was more active in the CQR lines of the transfectant parasites (Table 4) contrasts with what was observed in the field-derived strains, against which quinine was less active in the CQR parasites (Table 3 ). Both of these results are nevertheless consistent with previous studies; several fieldderived CQR strains have been shown to exhibit crossresistance to quinine in vitro, ${ }^{44}$ and the original characterization of the $\mathrm{C} 4^{\mathrm{Dd} 2}$ and $\mathrm{C} 6^{7 \mathrm{G} 8}$ lines revealed that these parasites are more sensitive to quinine and less susceptible to CQ than the $\mathrm{C} 2{ }^{\mathrm{GCO}}$ line. ${ }^{18}$ The reason for these conflicting patterns of 
quinine susceptibilities between transfectant and field-derived CQR parasites is not clear. The mode(s) of quinine action is not entirely understood, and it is likely that quinine targets one or more processes in addition to hemozoin formation. ${ }^{8}$ Moreover, resistance to quinine is a complex, multifactorial trait that remains largely unresolved ${ }^{43}$ but is currently thought to entail changes to PfCRT, PfMDR1, PfMRP1, and possibly other parasite proteins. ${ }^{11,15}$ Hence, when compared with the transgenic lines and CQS field-derived strains, the CQR field isolates may contain genetic differences (other than the changes to PfCRT) that decreased their susceptibility to quinine (but not to $\mathbf{1}$ and $\mathbf{6}$ ).

In addition, this study has uncovered important differences in how the two types of drugs interact with PfCRT ${ }^{\mathrm{CQR}}$ that may, at least in part, explain why the quinine dimers retain their activity in the CQR field strains. First, compounds 1 and 6 inhibited PfCRT ${ }^{\mathrm{CQR}}$ with 9-34 times the potency of quinine (Table 1). Second, quinine was a substrate of PfCRT ${ }^{\mathrm{CQR}}$ in situ, whereas the dimeric compounds were not (Figure 4a). The efflux of quinine via PfCRT ${ }^{\mathrm{CQR}}$, in the context of the other resistance-conferring changes that are likely to be present in the Dd2, P31 and FCB strains, could be sufficient to cause a marked reduction in the accumulation of quinine in the DV and hence a significant decrease in its antihemozoin and (relatively poor) anti-PfCRT ${ }^{\mathrm{CQR}}$ effects in these parasites. The absence of the "other" resistance-conferring changes in the CQR transfectant lines could shift the balance back toward quininesensitive status, perhaps because one or more routes of quinine influx into the DV are again operating and therefore augmenting its accumulation within the DV. By contrast, compounds $\mathbf{1}$ and $\mathbf{6}$ are high-affinity inhibitors of PfCRT ${ }^{\mathrm{CQR}}$ and are not effluxed by the protein, and as a consequence their antihemozoin and anti-PfCRT ${ }^{\mathrm{CQR}}$ activities may not be significantly affected by the presence or absence of other resistance-associated mutations, particularly if the extent to which these changes alter the DV accumulation of the dimers does not affect their antiplasmodial effects. Indeed, these other resistance-associated proteins may not even recognize or act upon the quinine dimers. Furthermore, it is worth noting that the field strains that were least susceptible to quinine (FCB and $\mathrm{P} 31$ ) both contain the $\mathrm{K} 1$ version of PfCRT ${ }^{\mathrm{CQR}}$, whereas the $\mathrm{Dd} 2$ strain (which carries the $\mathrm{Dd} 2$ version of PfCRT ${ }^{\mathrm{CQR}}$ ) was only slightly less sensitive to quinine than the CQS strain. It is therefore possible that the $\mathrm{K} 1$ form of PfCRT ${ }^{\mathrm{CQR}}$ has a greater capacity for quinine transport relative to the $\mathrm{Dd} 2$ (carried by the $\mathrm{C} 4^{\mathrm{Dd} 2}$ line) or $7 \mathrm{G} 8$ (carried by the $\mathrm{C} 6^{7 \mathrm{G} 8}$ line) versions of the protein, which would also contribute to the differences in quinine susceptibilities observed between the CQR lines and the FCB and P31 strains.

Compound 1 Was an Active Antimalarial in a Mouse Malaria Model. A preliminary in vivo evaluation of compounds 1 and 6 was performed using the Peters 4-day parasite suppression test in mice infected with a CQS strain of $P$. berghei. The antimalarial activity $\left(\mathrm{ED}_{50}, \mathrm{mg} / \mathrm{kg}\right)$ was determined for quinine (maximum dose $100 \mathrm{mg} / \mathrm{kg}$ ), 1 (maximum dose $120 \mathrm{mg} / \mathrm{kg}$ ) and 6 (maximum dose $50 \mathrm{mg} /$ $\mathrm{kg}$ ) administered orally once per day (Table 5). ${ }^{42,45}$ The overall health of the animals was monitored daily, and body weight was measured every second day. At the end of the test, the animals were found to have lost only around 5\% body weight and otherwise appeared normal (data not shown). The activity of compound $1\left(\mathrm{ED}_{50}\right.$ of $\left.38.2 \mathrm{mg} / \mathrm{kg}\right)$ was comparable to that of quinine $\left(\mathrm{ED}_{50}\right.$ of $\left.48.7 \mathrm{mg} / \mathrm{kg}\right)$. Compound 6 did not appear to
Table 5. In Vivo Antimalarial Activities of Compounds 1 and 6 in $P$. berghei-Infected Mice ${ }^{a}$

$\begin{array}{lccc}\text { compound } & \text { maximum dose }(\mathrm{mg} / \mathrm{kg}) & \mathrm{ED}_{50}(\mathrm{mg} / \mathrm{kg}) & \mathrm{CI}(95 \%) \\ \text { quinine } & 4 \times 100 & 48.7 & 37.4-63.3 \\ \mathbf{1} & 4 \times 120 & 38.2 & 21.9-66.8 \\ \mathbf{6} & 4 \times 50 & \begin{array}{c}\text { highly variable; suppression at } 50 \\ \mathrm{mg} / \mathrm{kg}\end{array}\end{array}$

${ }^{a} \mathrm{CD}-1$ mice were infected with $1 \times 10^{7}$ red blood cells infected with the CQS N-strain of $P$. berghei. Drug treatment at a range of concentrations up to the maximum dose indicated was initiated $2 \mathrm{~h}$ postinfection and continued for another 3 days. On day 4, parasitemia was measured by fluorescence staining of the infected red blood cells in tail-vein blood samples and compared with parasitemias of the untreated control group. Linear regression analysis of the data was performed to determine the effective dose at which $50 \%$ of parasitemia was cleared by the 4-day drug treatment $\left(\mathrm{ED}_{50}\right.$, in $\mathrm{mg}$ drug per $\mathrm{kg}$ mouse weight). CI: confidence interval.

exert an antimalarial effect when administered at doses between 10 and $40 \mathrm{mg} / \mathrm{kg}$, but was somewhat efficacious at $50 \mathrm{mg} / \mathrm{kg}$; data from two separate experiments yielded parasitemia suppression values of $15 \%$ and $83 \%$. This variability may have been due to greater weight loss in some of the animals after four drug doses of 6 . Given that weight loss can be a symptom of adverse physiological effects, further increases in the dose of 6 were not tried. Overcoming the in vivo toxicity of compound 6 will be the focus of future work that will aim to optimize this class of otherwise promising antimalarials.

Conclusions. This study describes the activities of a series of dimeric quinine compounds. These agents were found to be the most potent inhibitors of PfCRT ${ }^{\mathrm{CQR}}$ described to date with $\mathrm{IC}_{50}$ values between 1 and $5 \mu \mathrm{M}$ but are not themselves substrates of the transporter. Hence, unlike CQ and quinine, compounds 1 and $\mathbf{6}$ were not transported out of the DV of $\mathrm{CQR}$ parasites via PfCRT ${ }^{\mathrm{CQR}}$. Indeed, the observation that both compounds decreased the rate of (concanamycin Ainduced) alkalinization of the DV in both CQS and CQR parasites is consistent with both compounds accumulating to sufficiently high levels in the DV to exert a significant buffering effect. Compounds 1 and $\mathbf{6}$ inhibited the formation of synthetic hemozoin and were very effective antiplasmodials when tested in vitro against field- and laboratory-derived drug-resistant $P$. falciparum parasites. Compound $\mathbf{6}$ was also a potent reverser of $\mathrm{CQ}$ resistance in vitro, and the antimalarial activity of compound $\mathbf{1}$ was comparable to that of quinine when administered orally to mice infected with a CQS strain of $P$. berghei.

Of significant interest was the finding that compounds 1 and 6 are more active against CQR than CQS parasites and that this phenomenon is due, at least in part, to the presence of $\mathrm{PfCRT}^{\mathrm{CQR}}$ in the CQR stains. This result, together with the observations that the quinine dimers are potent inhibitors of $\mathrm{PfCRT}^{\mathrm{CQR}}$ in vitro and in situ, raises the possibility that the quinine dimers are also highly effective inhibitors of the transport of the (unknown) endogenous substrate(s) via PfCRT ${ }^{\mathrm{CQR}}$, and that this contributes to their antiplasmodial effect. The dimeric agents may therefore have multiple modes of action in CQR parasites. In addition to acting as resistance reversers (by blocking the $\mathrm{PfCRT}^{\mathrm{CQR}}$-mediated efflux of antimalarial drugs from the DV), the quinine dimers may target both (1) hemozoin formation and (2) the normal function of PfCRT ${ }^{\mathrm{CQR}}$. Given this potential trifunctionality and the relative ease and affordability of synthesizing the quinine 
dimers, these compounds could be administered in combination with a CQ-like drug to reverse CQ resistance as well as to achieve a combination therapy in which the two drugs inflict opposing selection forces on the CQ resistance mechanism. A paradigm shift toward considering PfCRT as a drug $\operatorname{target}^{11}$ and not just a key mediator of drug resistance could provide a framework for devising robust antimalarial strategies based on drug combinations that trap the parasite in a "resistance stalemate", with partner drugs chosen such that mutations required for resistance to one increase the parasite's sensitivity to the other drug.

\section{METHODS}

See Supporting Information for Supplementary Methods describing the synthesis of compounds $\mathbf{5}$ and $\mathbf{6}$, stability of the quinine dimers, culture of $P$. falciparum-infected red blood cells, in vitro parasite proliferation assays, and the 4-day Peters test.

Synthesis of Quinine Dimers 1, 2, 3, 4, and 7. Compounds 14 were synthesized as previously described. ${ }^{32}$ Compound 7 was synthesized from alkyne- and azide-modified quinine as previously described. $^{36}$

Measurements of CQ Transport in $X$. laevis Oocytes Expressing PfCRT. Expression of mutant and wild-type forms of PfCRT (from the strains Dd2 and D10, respectively) at the plasma membrane of $X$. laevis oocytes was performed as described previously. ${ }^{12}$ Briefly, oocytes were injected with cRNA encoding PfCRT (25 ng per oocyte) and the uptake of $\left[{ }^{3} \mathrm{H}\right] \mathrm{CQ}(0.3 \mu \mathrm{M} ; 20 \mathrm{Ci} /$ mmol, American Radiolabeled Chemicals) was measured 4-6 days postinjection using a method described previously. ${ }^{46}$ The influx measurements were made over $1-2 \mathrm{~h}$ at $27.5^{\circ} \mathrm{C}$ and in medium that contained $96 \mathrm{mM} \mathrm{NaCl}, 2 \mathrm{mM} \mathrm{KCl}, 1 \mathrm{mM} \mathrm{MgCl}, 1.8 \mathrm{mM} \mathrm{CaCl}_{2}, 10$ $\mathrm{mM}$ MES, $10 \mathrm{mM}$ Tris-base ( $\mathrm{pH}$ 6.0), and $15 \mu \mathrm{M}$ unlabeled CQ. Statistical comparisons were made with the Student's $t$-test for paired or unpaired samples or with ANOVA in conjunction with Tukey's multiple comparisons test.

Measurement of the DV $\mathrm{H}^{+}$Leak. Saponin-isolated trophozoitestage parasites containing the membrane-impermeant $\mathrm{pH}$-sensitive fluorescent indicator fluorescein-dextran (10000 MW; Life Technologies, Australia) in their DVs were prepared as described previously. ${ }^{25}$ The cells were suspended in a saline solution $(125 \mathrm{mM} \mathrm{NaCl}, 5 \mathrm{mM}$ $\mathrm{KCl}, 1 \mathrm{mM} \mathrm{MgCl} 2,20 \mathrm{mM}$ glucose, $25 \mathrm{mM}$ HEPES, $\mathrm{pH} 7.1$ ) at a density of $\sim 10^{7}$ cells $/ \mathrm{mL}$, and the $\mathrm{pH}$ of the DV was monitored at 37 ${ }^{\circ} \mathrm{C}$ using a PerkinElmer Life Sciences LS50B fluorometer with a dual excitation Fast Filter accessory (excitation 490 and $450 \mathrm{~nm}$; emission $520 \mathrm{~nm}$ ). The experiments entailed monitoring the alkalinization of the DV upon addition of the $\mathrm{V}$-type $\mathrm{H}^{+}$-ATPase inhibitor concanamycin A $(100 \mathrm{nM})$, in the presence and absence of the drugs of interest. Half-times for DV alkalinization were calculated as outlined elsewhere. ${ }^{23}$

Inhibition of $\beta$-Hematin Formation. Inhibition of the formation of $\beta$-hematin in vitro was measured using a colorimetric assay based on the pyridine ferrihemochrome method. ${ }^{39}$ Briefly, aqueous solutions of dimers and hematin were mixed to give final concentrations of $0-10$ equiv of dimer relative to hematin. A prewarmed supersaturated solution of sodium acetate trihydrate $\left(60^{\circ} \mathrm{C}\right)$ was added, and the samples were incubated at $60^{\circ} \mathrm{C}$ for $1 \mathrm{~h}$. The reactions were quenched at RT, and the $\beta$-hematin was allowed to settle at RT for $20 \mathrm{~h}$. The supernatant of each sample was transferred to a cuvette, and the absorbance was read at $405 \mathrm{~nm}$. The $\mathrm{IC}_{50}$ values were derived from curves fitted to the resulting dose-response data (GraphPad Prism).

\section{ASSOCIATED CONTENT}

\section{S Supporting Information}

Supplementary methods. This material is available free of charge via the Internet at http://pubs.acs.org.

\section{AUTHOR INFORMATION}

\section{Corresponding Authors}

*E-mail: hrycyna@purdue.edu.

*E-mail: rowena.martin@anu.edu.au.

*E-mail: chml@purdue.edu.

\section{Author Contributions}

J.C. and R.E.M. contributed equally to this work and are joint senior authors. C.A.H., R.L.S., and A.M.L. contributed equally to this work and are co-first authors.

\section{Notes}

The authors declare no competing financial interest.

\section{ACKNOWLEDGMENTS}

We thank E. Baker for technical assistance and the Australian Red Cross Blood Service for the provision of blood. This work was supported by a grant from the Bill \& Melinda Gates Foundation Grand Challenges Exploration Program (to C.A.H. and J.C.), Grants 471472 and 1007035 from the Australian National Health and Medical Research Council (NHMRC; to R.E.M.) and Grants R01 AI50234 and R01 AI079709 from the US National Institutes of Health (to D.A.F.). R.E.M. was supported by NHMRC fellowships 520320 and 1053082 and the L'Oréal Australia For Women in Science program. A.M.L. was supported by a NHMRC Overseas Biomedical Fellowship (585519).

\section{REFERENCES}

(1) WHO. (2012) World malaria report 2012, p 200.

(2) Trape, J. F. (2001) The public health impact of chloroquine resistance in Africa. Am. J. Trop. Med. Hyg. 64, 12-17.

(3) Petersen, I., Eastman, R., and Lanzer, M. (2011) Drug-resistant malaria: Molecular mechanisms and implications for public health. FEBS Lett. 585, 1551-1562.

(4) Dondorp, A. M., Nosten, F., Yi, P., Das, D., Phyo, A. P., Tarning, J., Lwin, K. M., Ariey, F., Hanpithakpong, W., Lee, S. J., Ringwald, P., Silamut, K., Imwong, M., Chotivanich, K., Lim, P., Herdman, T., An, S. S., Yeung, S., Singhasivanon, P., Day, N. P., Lindegardh, N., Socheat, D., and White, N. J. (2009) Artemisinin resistance in Plasmodium falciparum malaria. N. Engl. J. Med. 361, 455-467.

(5) Phyo, A. P., Nkhoma, S., Stepniewska, K., Ashley, E. A., Nair, S., McGready, R., ler Moo, C., Al-Saai, S., Dondorp, A. M., Lwin, K. M., Singhasivanon, P., Day, N. P., White, N. J., Anderson, T. J., and Nosten, F. (2012) Emergence of artemisinin-resistant malaria on the western border of Thailand: A longitudinal study. Lancet 379, 19601966.

(6) Bray, P. G., Mungthin, M., Hastings, I. M., Biagini, G. A., Saidu, D. K., Lakshmanan, V., Johnson, D. J., Hughes, R. H., Stocks, P. A., O'Neill, P. M., Fidock, D. A., Warhurst, D. C., and Ward, S. A. (2006) PfCRT and the trans-vacuolar proton electrochemical gradient: Regulating the access of chloroquine to ferriprotoporphyrin IX. Mol. Microbiol. 62, 238-251.

(7) Egan, T. J. (2008) Haemozoin formation. Mol. Biochem. Parasitol. 157, 127-136.

(8) Fitch, C. D. (2004) Ferriprotoporphyrin IX, phospholipids, and the antimalarial actions of quinoline drugs. Life Sci. 74, 1957-1972.

(9) Sanchez, C. P., Mayer, S., Nurhasanah, A., Stein, W. D., and Lanzer, M. (2011) Genetic linkage analyses redefine the roles of PfCRT and PfMDR1 in drug accumulation and susceptibility in Plasmodium falciparum. Mol. Microbiol. 82, 865-878.

(10) Cooper, R. A., Ferdig, M. T., Su, X. Z., Ursos, L. M., Mu, J., Nomura, T., Fujioka, H., Fidock, D. A., Roepe, P. D., and Wellems, T. E. (2002) Alternative mutations at position 76 of the vacuolar transmembrane protein PfCRT are associated with chloroquine resistance and unique stereospecific quinine and quinidine responses in Plasmodium falciparum. Mol. Pharmacol. 61, 35-42. 
(11) Summers, R. L., Nash, M. N., and Martin, R. E. (2012) Know your enemy: Understanding the role of PfCRT in drug resistance could lead to new antimalarial tactics. Cell. Mol. Life Sci. 69, 19671995.

(12) Martin, R. E., Marchetti, R. V., Cowan, A. I., Howitt, S. M., Broer, S., and Kirk, K. (2009) Chloroquine transport via the malaria parasite's chloroquine resistance transporter. Science 325, 1680-1682.

(13) Cooper, R. A., Lane, K. D., Deng, B., Mu, J., Patel, J. J., Wellems, T. E., Su, X., and Ferdig, M. T. (2007) Mutations in transmembrane domains 1,4 and 9 of the Plasmodium falciparum chloroquine resistance transporter alter susceptibility to chloroquine, quinine and quinidine. Mol. Microbiol. 63, 270-282.

(14) Sidhu, A. B., Valderramos, S. G., and Fidock, D. A. (2005) pfmdrl mutations contribute to quinine resistance and enhance mefloquine and artemisinin sensitivity in Plasmodium falciparum. Mol. Microbiol. 57, 913-926.

(15) Mu, J., Ferdig, M. T., Feng, X., Joy, D. A., Duan, J., Furuya, T., Subramanian, G., Aravind, L., Cooper, R. A., Wootton, J. C., Xiong, M., and Su, X. Z. (2003) Multiple transporters associated with malaria parasite responses to chloroquine and quinine. Mol. Microbiol. 49, 977-989.

(16) Fidock, D. A., Nomura, T., Talley, A. K., Cooper, R. A., Dzekunov, S. M., Ferdig, M. T., Ursos, L. M., Sidhu, A. B., Naude, B., Deitsch, K. W., Su, X. Z., Wootton, J. C., Roepe, P. D., and Wellems, T. E. (2000) Mutations in the P. falciparum digestive vacuole transmembrane protein PfCRT and evidence for their role in chloroquine resistance. Mol. Cell 6, 861-871.

(17) Ecker, A., Lehane, A. M., Clain, J., and Fidock, D. A. (2012) PfCRT and its role in antimalarial drug resistance. Trends Parasitol. 28, 504-514.

(18) Sidhu, A. B., Verdier-Pinard, D., and Fidock, D. A. (2002) Chloroquine resistance in Plasmodium falciparum malaria parasites conferred by pfcrt mutations. Science 298, 210-213.

(19) Martin, R. E., and Kirk, K. (2004) The malaria parasite's chloroquine resistance transporter is a member of the drug/metabolite transporter superfamily. Mol. Biol. Evol. 21, 1938-1949.

(20) Johnson, D. J., Fidock, D. A., Mungthin, M., Lakshmanan, V., Sidhu, A. B., Bray, P. G., and Ward, S. A. (2004) Evidence for a central role for PfCRT in conferring Plasmodium falciparum resistance to diverse antimalarial agents. Mol. Cell 15, 867-877.

(21) Waller, K. L., Muhle, R. A., Ursos, L. M., Horrocks, P., VerdierPinard, D., Sidhu, A. B., Fujioka, H., Roepe, P. D., and Fidock, D. A. (2003) Chloroquine resistance modulated in vitro by expression levels of the Plasmodium falciparum chloroquine resistance transporter. J. Biol. Chem. 278, 33593-33601.

(22) Sanchez, C. P., McLean, J. E., Stein, W., and Lanzer, M. (2004) Evidence for a substrate specific and inhibitable drug efflux system in chloroquine resistant Plasmodium falciparum strains. Biochemistry 43, 16365-16373.

(23) Lehane, A. M., Hayward, R., Saliba, K. J., and Kirk, K. (2008) A verapamil-sensitive chloroquine-associated $\mathrm{H}^{+}$leak from the digestive vacuole in chloroquine-resistant malaria parasites. J. Cell Sci. 121, 1624-1632.

(24) Lehane, A. M., and Kirk, K. (2008) Chloroquine resistanceconferring mutations in $p f c r t$ give rise to a chloroquine-associated $\mathrm{H}^{+}$ leak from the malaria parasite's digestive vacuole. Antimicrob. Agents Chemother. 52, 4374-4380.

(25) Lehane, A. M., and Kirk, K. (2010) Efflux of a range of antimalarial drugs and 'chloroquine resistance reversers' from the digestive vacuole in malaria parasites with mutant PfCRT. Mol. Microbiol. 77, 1039-1051.

(26) Sanchez, C. P., Stein, W. D., and Lanzer, M. (2008) Dissecting the components of quinine accumulation in Plasmodium falciparum. Mol. Microbiol. 67, 1081-1093.

(27) Burgess, S. J., Selzer, A., Kelly, J. X., Smilkstein, M. J., Riscoe, M. K., and Peyton, D. H. (2006) A chloroquine-like molecule designed to reverse resistance in Plasmodium falciparum. J. Med. Chem. 49, 56235625 .
(28) Zishiri, V. K., Joshi, M. C., Hunter, R., Chibale, K., Smith, P. J., Summers, R. L., Martin, R. E., and Egan, T. J. (2011) Quinoline antimalarials containing a dibemethin group are active against chloroquinone-resistant Plasmodium falciparum and inhibit chloroquine transport via the $P$. falciparum chloroquine-resistance transporter (PfCRT). J. Med. Chem. 54, 6956-6968.

(29) Ubben, D., and Poll, E. M. (2013) MMV in partnership: The Eurartesim(R) experience. Malar. J. 12, 211.

(30) Briolant, S., Henry, M., Oeuvray, C., Amalvict, R., Baret, E., Didillon, E., Rogier, C., and Pradines, B. (2010) Absence of association between piperaquine in vitro responses and polymorphisms in the pfcrt, pfmdr1, pfmrp, and pfnhe genes in Plasmodium falciparum. Antimicrob. Agents Chemother. 54, 3537-3544.

(31) Hao, M., Jia, D., Li, Q., He, Y., Yuan, L., Xu, S., Chen, K., Wu, J., Shen, L., Sun, L., Zhao, H., Yang, Z., and Cui, L. (2013) In vitro sensitivities of Plasmodium falciparum isolates from the ChinaMyanmar border to piperaquine and association with polymorphisms in candidate genes. Antimicrob. Agents Chemother. 57, 1723-1729.

(32) Pires, M. M., Emmert, D., Hrycyna, C. A., and Chmielewski, J. (2009) Inhibition of P-glycoprotein-mediated paclitaxel resistance by reversibly linked quinine homodimers. Mol. Pharmacol. 75, 92-100.

(33) Pires, M. M., Hrycyna, C. A., and Chmielewski, J. (2006) Bivalent probes of the human multidrug transporter P-glycoprotein. Biochemistry 45, 11695-11702.

(34) Wong, I. L., Chan, K. F., Tsang, K. H., Lam, C. Y., Zhao, Y., Chan, T. H., and Chow, L. M. (2009) Modulation of multidrug resistance protein 1 (MRP1/ABCC1)-mediated multidrug resistance by bivalent apigenin homodimers and their derivatives. J. Med. Chem. 52, 5311-5322.

(35) Brunner, H., Bügler, J., and Nuber, B. (1995) Enantioselective catalysis 98. Preparation of 9-amino(9-deoxy) cinchona alkaloids. Tetrahedron: Asymmetry 6, 1699-1702.

(36) Kuriakose, J., Hrycyna, C. A., and Chmielewski, J. (2012) Click chemistry-derived bivalent quinine inhibitors of P-glycoproteinmediated cellular efflux. Bioorg. Med. Chem. Lett. 22, 4410-4412.

(37) Martin, R. E., Butterworth, A. S., Gardiner, D. L., Kirk, K., McCarthy, J. S., and Skinner-Adams, T. S. (2012) Saquinavir inhibits the malaria parasite's chloroquine resistance transporter. Antimicrob. Agents Chemother. 56, 2283-2289.

(38) Pires, M. M., and Chmielewski, J. (2008) Fluorescence imaging of cellular glutathione using a latent rhodamine. Org. Lett. 10, 837840.

(39) Ncokazi, K. K., and Egan, T. J. (2005) A colorimetric highthroughput beta-hematin inhibition screening assay for use in the search for antimalarial compounds. Anal. Biochem. 338, 306-319.

(40) Desjardins, R. E., Canfield, C. J., Haynes, J. D., and Chulay, J. D. (1979) Quantitative assessment of antimalarial activity in vitro by a semiautomated microdilution technique. Antimicrob. Agents Chemother. $16,710-718$.

(41) Fidock, D. A., Nomura, T., and Wellems, T. E. (1998) Cycloguanil and its parent compound proguanil demonstrate distinct activities against Plasmodium falciparum malaria parasites transformed with human dihydrofolate reductase. Mol. Pharmacol. 54, 1140-1147.

(42) Fidock, D. A., Rosenthal, P. J., Croft, S. L., Brun, R., and Nwaka, S. (2004) Antimalarial drug discovery: Efficacy models for compound screening. Nat. Rev. Drug Discovery 3, 509-520.

(43) Ferdig, M. T., Cooper, R. A., Mu, J., Deng, B., Joy, D. A., Su, X. Z., and Wellems, T. E. (2004) Dissecting the loci of low-level quinine resistance in malaria parasites. Mol. Microbiol. 52, 985-997.

(44) Valderramos, S. G., Valderramos, J. C., Musset, L., Purcell, L. A., Mercereau-Puijalon, O., Legrand, E., and Fidock, D. A. (2010) Identification of a mutant PfCRT-mediated chloroquine tolerance phenotype in Plasmodium falciparum. PLoS Pathog. 6, No. e1000887.

(45) Peters, W., Robinson, B. L., and Ellis, D. S. (1987) The chemotherapy of rodent malaria. XLII. Halofantrine and halofantrine resistance. Ann. Trop. Med. Parasitol. 81, 639-646.

(46) Bröer, S. (2003) Xenopus laevis oocytes. Methods Mol. Biol. 227, $245-258$. 